\title{
ARQUITECTURA Y SOCIEDAD
}

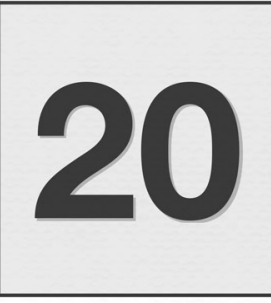

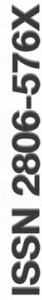

E D I C I Ó N

VOL. 1 JULIO-DICIEMBRE-2021

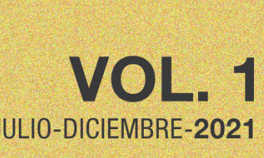

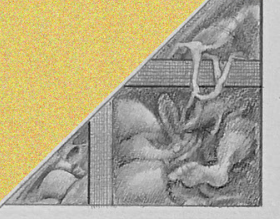
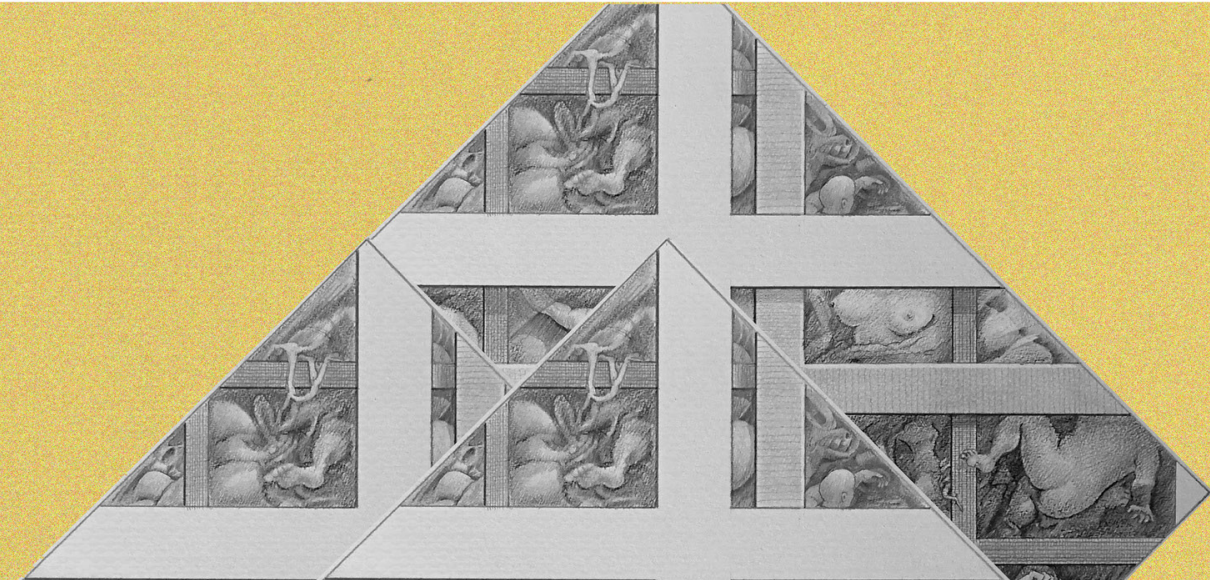

yea $\Rightarrow$ bea
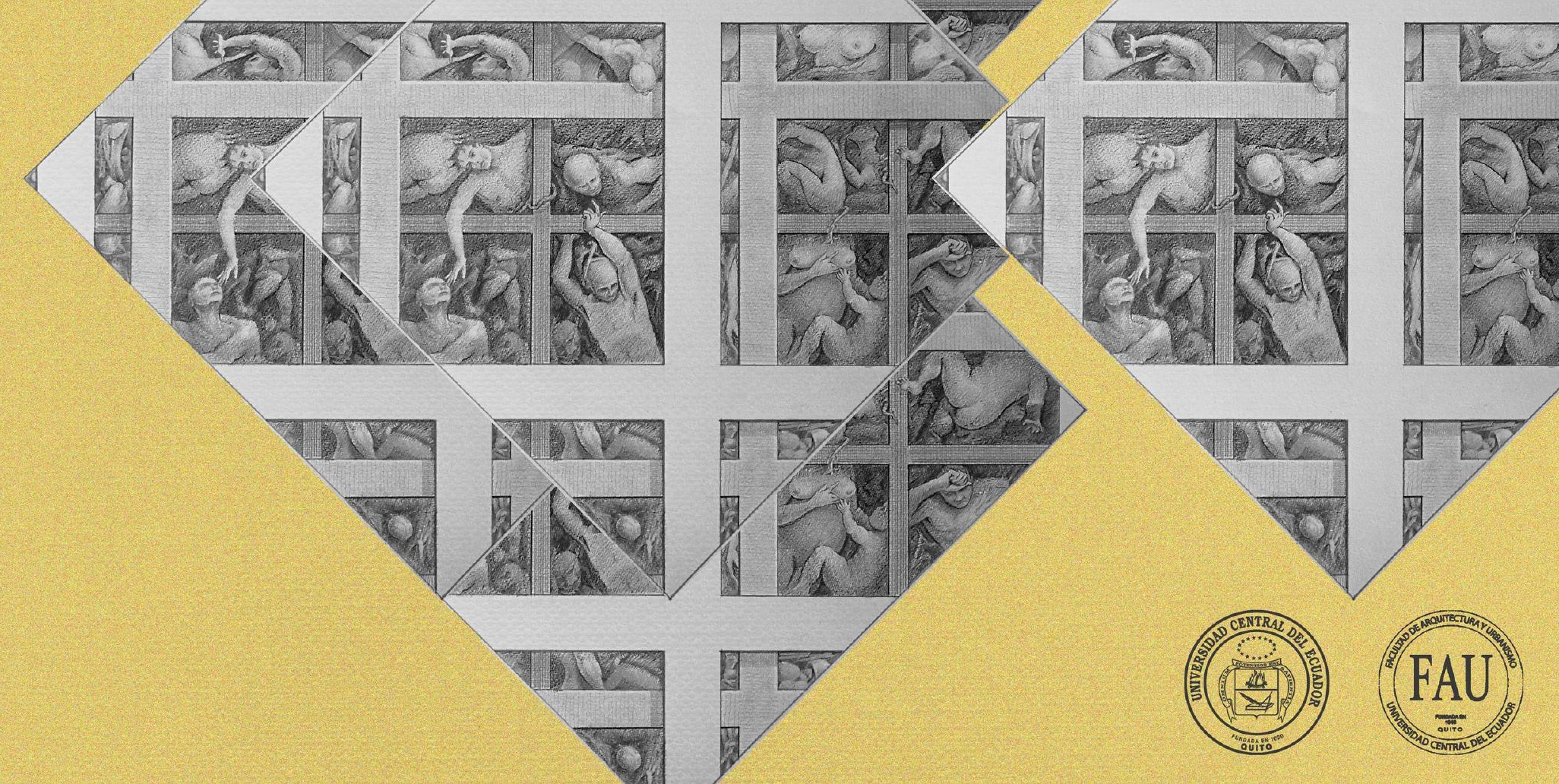


\section{Arquitectura y Sociedad}

Facultad de Arquitectura y Urbanismo

Universidad Central del Ecuador

Maritza Balcázar Basantes

Decana

Kléver Vásquez Vargas

Director editorial

\section{Comité editorial}

Andrea Salazar Veloz

Kléver Vásquez Vargas

\section{Comité científico}

María José Freire Silva

María Isabel Carrasco Vintimilla

Fernando Puente Sotomayor

Gina Maestre Góngora

Marco Salazar Valle

Ángela Díaz Márquez

Gonzalo Hoyos Bucheli

Manuel Martín Hernández

Fernanda Aguirre

Luis Buitrón Aguas

Carlos Crespo Sánchez

Carla García

Fernando Huanca

Janaina Marx

Verónica Rosero

Esteban Zalamea

Carla Maranguello

Universidad Central del Ecuador

Universidad del Azuay

Universidad Central del Ecuador

Universidad Cooperativa de Colombia

Universidad Central del Ecuador

Universidad de Las Américas

Universidad Internacional SEK

Universidad de Guadalajara

Universidad del Azuay

Universidad Central del Ecuador

Universidad de Guadalajara

Universidad de Buenos Aires

Universidad Internacional del Ecuador - Loja

Universidad Central del Ecuador

Universidad Central del Ecuador

Universidad de Cuenca

Universidad de Buenos Aires 
Imagen de portada

"Estructura profunda" Kléver Vásquez

\section{Diseño y diagramación}

Nicolás Sánchez

\section{Colaboración}

Bélgica Toapanta

\section{Correspondencia}

Arquitectura y Sociedad

https://revistadigital.uce.edu.ec/index. php/arquitectura

FAU editorial

Correo electrónico

fau.editorial@uce.edu.ec

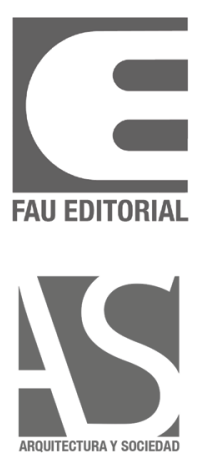




\section{DECONSTRUYENDO EL CONCEPTO DE SMART CITY}

Una mirada crítica a su aplicación en latinoamérica.

\section{DECONSTRUCTING THE CONCEPT OF SMART CITY}

A critical look at its application in Latin America

DOl: https://doi.org/10.29166/ays.v1i20.3497

Fecha de recepción: 16/06/2021.

Fecha de aceptación: 21/09/2021

1.Magíster en Arte, mención en Teoría e Historia del Arte. Universidad de Chile. Arquitecto, coordinador y docente de pregrado Facultad de Ingeniería, Arquitectura y Paisaje. Universidad Central de Chile.

Correo: eferrerr@ucentral.cl

2. Magíster en Arquitectura, Pontificia Universidad Católica de Chile. Arquitecta y docente de pregrado Facultad de Ingeniería, Arquitectura y Paisaje. Universidad Central de Chile.

Correo: carolinacastillobutting@gmail.com 


\section{Resumen:}

Con el desarrollo de las tecnologías de la información y la comunicación, así como aquellas tecnologías relacionadas con nuevas fuentes energéticas y las políticas de gestión ambiental urbana, que ya llevan varios años, no resulta extraño que se haya propiciado el concepto de "Smart City" (Ciudad Inteligente). Existen varias nuevas "ciudades inteligentes" en el mundo y se enmarcan, en general, en realizaciones de alto estándar en países avanzados, pero también se aplica como índice de medición de ciudades existentes, tanto al interior de un país, como ranking entre ciudades de diferentes países del mundo. Sin embargo, también es posible entenderlo como iniciativa de desarrollo comunitario en localidades existentes, así como estrategia para proyectos utópicos. Todas estas posibilidades indican que no tiene un significado unívoco, que pueda ser concebido simplemente como la aplicación de tecnologías avanzadas en la gestión de la ciudad, más bien se trata de un concepto complejo de contornos ideológicos, que tiene derivaciones y aplicaciones importantes para la vida urbana contemporánea.

Este artículo analiza las modalidades e implicancias del concepto en el contexto latinoamericano, por lo cual se ha privilegiado el componente social sobre otras consideraciones. A través del análisis de una serie de documentos y experiencias producidas principalmente entre los años 2015 y 2021, se aborda una perspectiva crítica del concepto "Smart City", como un aporte al debate para quienes estudian la evolución de las ciudades latinoamericanas en el futuro próximo.

\section{Abstract:}

With the development of information and communication technologies, as well as those technologies related to new energy sources and urban environmental management policies, which have been going on for several years now, it is not surprising that the concept of "Smart City" has been promoted. There are several new smart cities in the world and they are framed, in general, in high-standard realizations in advanced countries, but it is also applied as an index of measurement of existing cities, both within a country, as a ranking between cities in different countries of the world. However, it is also possible to understand it as a community development initiative in existing localities, as well as a strategy for utopian projects. All these possibilities indicate that it does not have a univocal meaning, that it can be conceived simply as the application of advanced technologies in the management of the city, rather it is a complex concept of ideological contours, which has important derivations and applications for life contemporary urban.

This article analyzes the modalities and implications of the concept in the Latin American context, for which the social component has been privileged over other considerations. Through the analysis of a series of documents and experiences produced mainly between the years 2015 and 2021, a critical perspective of the "Smart City" concept is approached, as a contribution to the debate for those who study the evolution of Latin American cities in the near future.
Palabras clave: Smart City, neoliberalismo, tecnología ciudadanía, desigualdad social

Keywords: Smart City, neoliberalism, technology, citizenship, social inequality. 


\section{Introducción}

Corría el año 1997 y el Foro Mundial de "Ciudades Inteligentes" predijo que habría para 2007, más de 50.000 "ciudades inteligentes" en todo el mundo. Si bien este pronóstico no llego a materializarse, los proyectos de "ciudades inteligentes" no han desaparecido y hoy existe un gran debate en torno al término y su aplicación, multiplicándose las conferencias, debates, informes, boletines y consultorías acerca de "ciudades inteligentes" alrededor del planeta. Junto al debate, se ha incrementado también la inversión en "ciudades inteligentes". Según Saunders y Baeck (2015), el mercado para las "ciudades inteligentes" tuvo un valor de $\$ 8.8$ mil millones en 2014 y una proyección para el mercado global de tecnologías y servicios de "ciudades inteligentes", por un valor de \$ 408 mil millones para 2020.

Bajo esta premisa, nace la pregunta acerca de cuál es la visión del término "Smart City" en nuestra región. Las ciudades latinoamericanas, están lejos aún de paradigmas inteligentes tales como Songdo en Corea del Sur, en dónde ya viven 180.000 mil personas en una isla artificial que comenzó desde cero el año 2003 (Orgaz, 2021). Tras varios años de pilotos en China y la India, y un sin número de aplicaciones en países europeos en ciudades ya existentes, en el debate global ya comienzan a sentirse las críticas y dudas respecto del avance en la utilización de tecnologías de vanguardia, para gestión, control y monitoreo de ciudades. Estas voces, proponen un avance hacia modelos mas humanos y colaborativos de desarrollo, en donde las tecnologías involucren directamente a los ciudadanos e impacten en la cotidianeidad real de las personas.

La investigación, coloca en el debate la idea de un término que aún sigue abierto y lleno de contradicciones en nuestro contexto, por lo que se hace necesario revisar e indagar en sus orígenes epistemológicos para direccionar su avance y sus múltiples posibilidades. No existe una definición unívoca del término, como señala Bauer. "There is no unique and standardized definition of a "Smart City", although some are started to be proposed; the same is true for the term smart region" (Bauer, et al., 2019: 1).

Este trabajo da cuenta del uso que el concepto ha tenido en diversos e importantes espacios latinoamericanos tanto gubernamentales, no gubernamentales como académicos. Si bien se puede afirmar que Latinoamérica es más receptora que productora de tecnologías, el concepto de "Smart City" no pertenece exclusivamente al orden tecno-científico, sino al de un conjunto heterogéneo de componentes desde lo económico hasta lo social. Es justamente el punto de partida desde donde surgen las interpretaciones, apropiaciones y contradicciones, puesto que diferentes lugares de enunciación y sus posiciones ideológicas definen, enfatizan u omiten componentes de acuerdo a determinadas visiones del desarrollo social y urbano.

En este sentido este trabajo analiza diversas posiciones y etapas que el concepto ha tenido y cómo se ha estado utilizando y debatiendo en importantes espacios latinoamericanos.

En el apartado "Resultados" se proponen y exponen las categorías narrativas que se han obtenido del análisis teórico 0 conceptual y que permiten un análisis conceptual del concepto "Smart City", mientras que en el apartado "Discusión" se debaten los alcances y perspectivas críticas de esas narrativas, sin que deban ser consideradas necesariamente como conclusiones, sino como aperturas para desarrollos que se encuentran actualmente abiertos. 


\section{Metodología}

La metodología utilizada consiste en el análisis de tipo teórico o conceptual en base al examen de una selección de documentos de variadas fuentes (informes oficiales, modelos de medición, ponencias y artículos), que dan cuenta de diferentes discursividades o narrativas respecto del concepto de "Smart City", especialmente de textos producidos entre 2015 y 2021. Esta selección está orientada a pesquisar el sentido dado al concepto de "Smart City" en diversos espacios en latinoamérica, por lo cual la mayor parte de las fuentes procede de autores latinoamericanos, no obstante, se consideran autores de otras regiones por el interés que tienen para efectos del análisis.

\section{Análisis de fuentes}

El punto de partida de este análisis es el informe "Rethinking smart cities from the ground up" (Saunders, Baeck, 2015), documento que enuncia cambios paradigmáticos y sienta precedentes en Ios planes piloto actuales de "ciudades inteligentes”. El rango del análisis concluye con el reciente evento internacional "Valdivia Urban Lab" realizado en Chile en mayo de 2021. En este rango se han examinado una serie de documentos seleccionados por su aporte a la discusión del tema tratado. Este trabajo de una primera selección se grafica en la figura 1, del cual se obtienen 3 tipos de fuentes que se han clasificado en: Modelo e indicadores; casos de estudio; diagnóstico, debate y concepto. En la figura 2 se indican los porcentajes de incidencia en cada tipo de fuente.

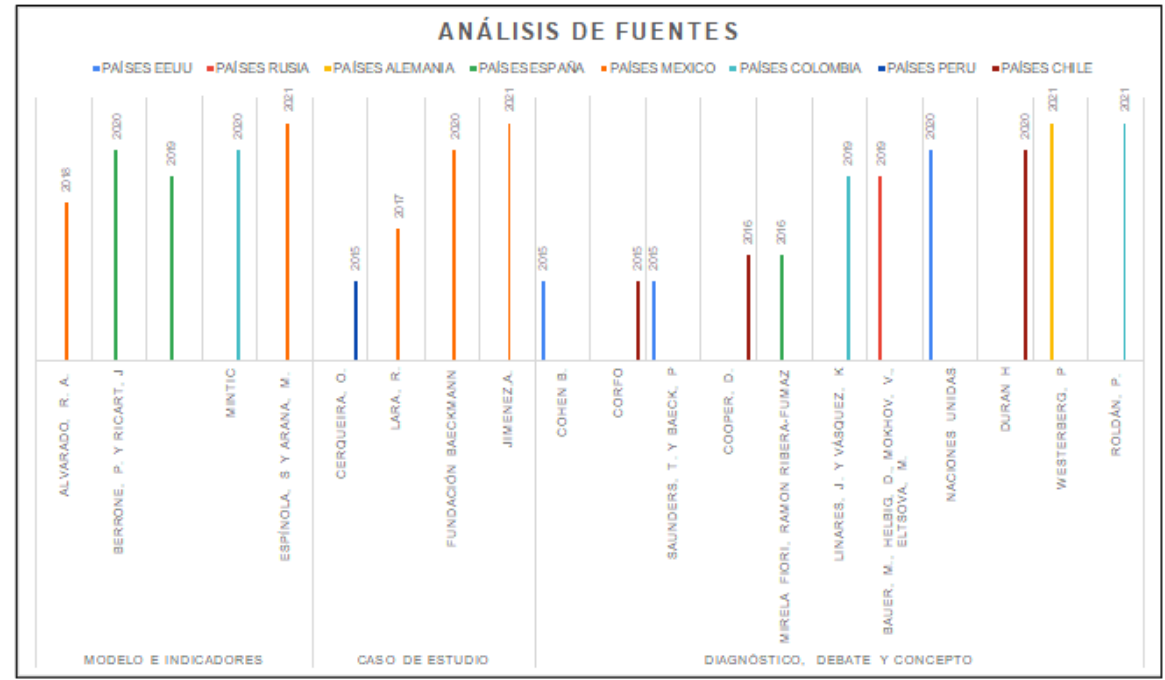

Figura 1. Análisis de fuentes. Fuente: Elaboración Propia en base a las fuentes analizadas.

\section{Tipo de Fuente Analizada (2015-2021)}

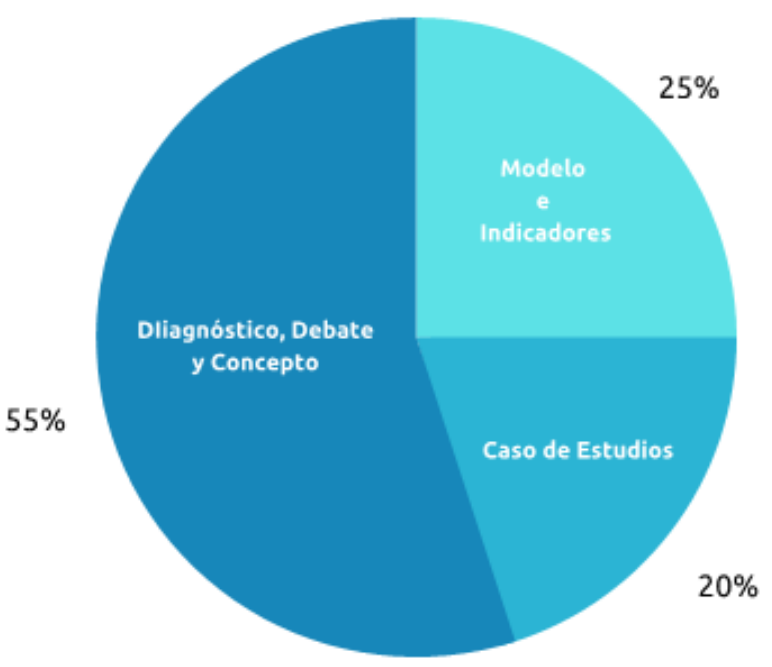

Figura 2. Tipo de fiente analzada (2015-2021) Fuente: Flaboración Propia en base a las fuentes analizadas. 


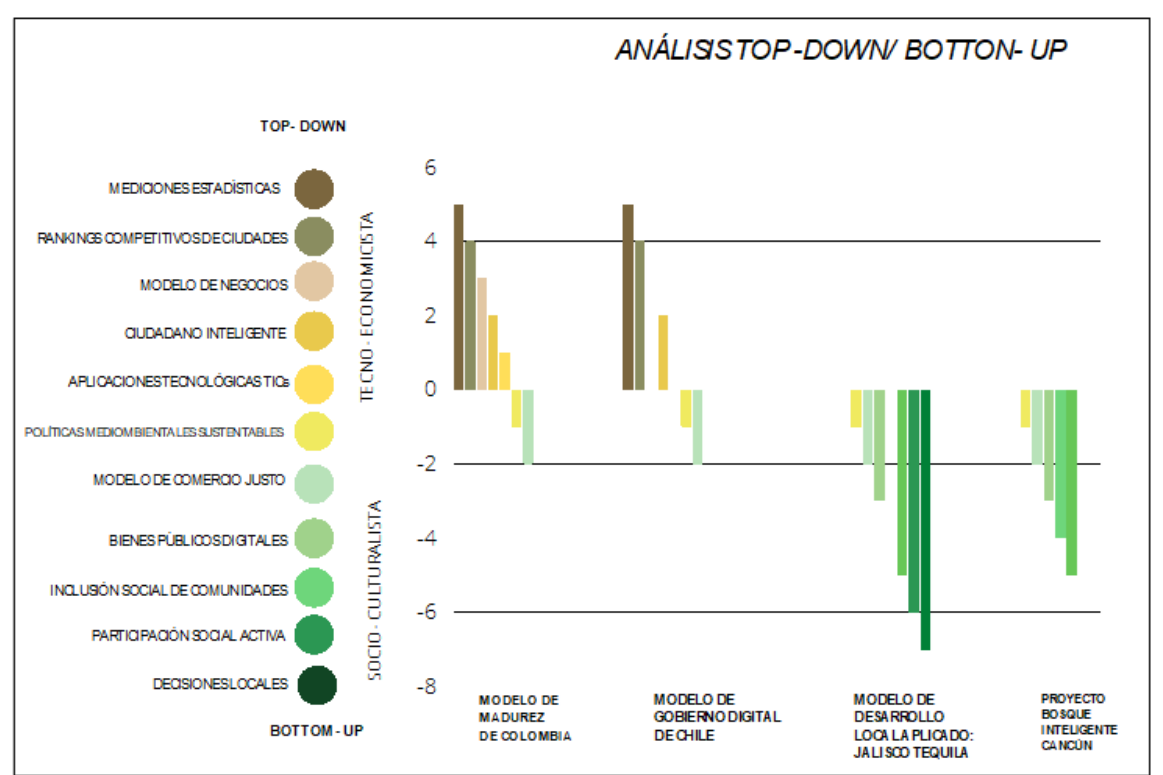

Figura 3. Comparación modelos

Fuente: Elaboración Propia en base a las fuentes analizadas.

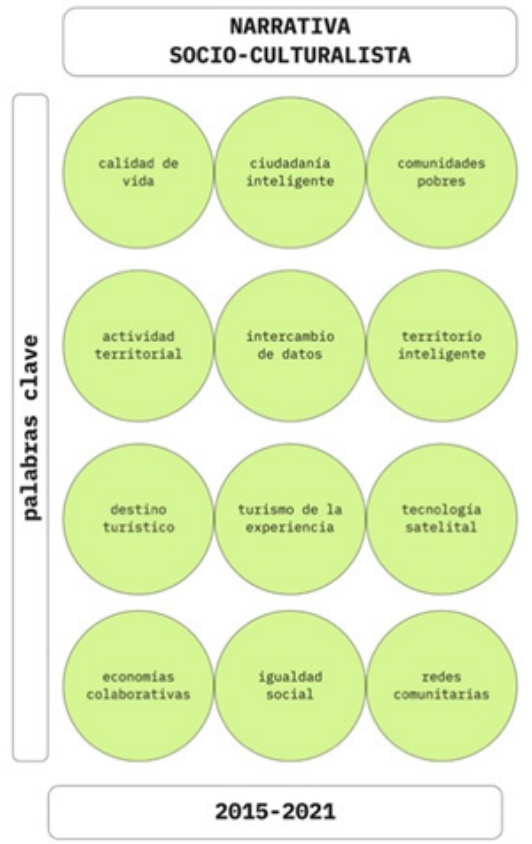

Figura 4. Comparación de narrativas en base a ideas clave. Fuente: Elaboración Propia en base a las fuentes analizadas.

\section{Línea de investigación}

El principal criterio de análisis de textos ha consistido en el factor social del concepto "Smart City". Si bien el concepto contiene varios componentes tales como medioambiente, transporte 0 fuentes energéticas, se ha priorizado el componente 0 factor social por la relevancia que tiene para las políticas públicas del sub-continente. Este factor se entiende como la importancia, relevancia, exclusión, exclusión o limitaciones que se otorga a la participación social de los habitantes de las localidades donde se implementa 0 aplica algún indicador, programa o proyecto de "Smart City".

La línea de investigación que se ha seguido consiste en la pregunta por los límites conceptuales del término "Smart City", la elasticidad de sus significados y las narrativas asociadas a distintos lugares de enunciación.

En este sentido, se ha operado en base a un modelo "Top-Down / Bottom-Up", (desde arriba-hacia abajo / Desde abajo-hacia arriba) para caracterizar y clasificar los diferentes documentos seleccionados, según el tratamiento dado al factor social (véase más adelante en la figura 3).

Los problemas sociales endémicos relacionados con la marginalidad, la falta de oportunidades, la desigualdad social, las limitaciones al acceso de bienes públicos, entre otros conflictos de la región, agudizados en el contexto de desarrollo socioeconómico del capitalismo globalizado, justifican una mirada desde este punto de vista al concepto "Smart City".

Así mismo el propio concepto "Smart City" está siendo tratado con creciente atención y reiterada incidencia en los espacios académicos ligados a la arquitectura y los estudios urbanos, no obstante, los usos y contenidos diversificados, discordantes 0 antinómicos que se asocian al concepto, 
requieren en sí mismo análisis teóricoconceptuales de los cuales este estudio participa.

Cabe agregar que el concepto "Smart City" tiene conceptos relacionados 0 derivaciones como territorio inteligente, pueblo inteligente, localidad o región inteligente, pero debido a los alcances del estudio no se han establecido distinciones específicas para estas diferencias escalares.

\section{Resultados}

Mediante la selección de fuentes consideradas relevantes en determinados espacios latinoamericanos para el concepto de "Smart City" (por ejemplo, instituciones gubernamentales, organizaciones no gubernamentales y artículos de tipo académico), se procedió, en una primera instancia, a caracterizarlos según sus posiciones de enunciación en función del factor social (véase la figura 1). Con independencia de la fecha de producción de documentos sobre "Smart Cities", se advierte que el factor social, cualquiera sea su acepción o significado, ha estado presente en forma continua. Si bien, para ciertos autores como Cohen (Cohen,

2015) el concepto ha evolucionado en el tiempo (Smart-Cities 2.00 3.0, etc.), lo cual explicaría la aparición de narrativas diferentes y la relevancia en etapas actuales del factor social, la presencia de este factor a través del tiempo permite afirmar que diferentes narrativas se deben más bien a posicionamientos ideológicos, que enfatizan ciertos componentes o relevan determinadas categorías por sobre otras, más que a una evolución natural del concepto "Smart City" en el tiempo.

Lo anterior exigió establecer una categorización sincrónica y no diacrónica del concepto, en base a una segunda selección de documentos (en función de su carácter ejemplificador), lo cual permitió establecer y aplicar un modelo "Top-Down / Bottom-

Up", donde se eligieron los siguientes documentos: Modelo de Madurez de Colombia; Modelo de Gobierno digital de Chile; Modelo de Desarrollo Local Aplicado en Jalisco Tequila; Proyecto Bosque Inteligente Cancún. Este último se incluye como una nueva variante, pero por los escasos antecedentes disponibles, sólo se menciona tangencialmente en este artículo.

Este análisis clasificatorio se grafica en la figura 3, donde los principales componentes significantes del concepto "Smart City" se han organizado en un diagrama "TopDown / Bottom-Up", donde el significante "Aplicaciones tecnológicas Tic's" correponde al nivel cero 0 "Middle". En términos teórico-conceptuales se ha asociado el componente "Top-Down" con la narrativa denominada tecno-economicista, mientras que el componente "Bottom-Up" se ha asociado a la narrativa "socio-culturalista". La denominación "narrativa", utilizada para caracterizar el resultado obtenido, se asocia al alcance discursivo ideológico del análisis. Lo anterior se justifica al comparar las palabras clave contenidas en los diferentes documentos clasificados según narrativas, lo cual se grafica en la figura 4. Por lo tanto, aquellos discursos que priorizan el mundo de los negocios, el gobierno central, los rankings competitivos y las mediciones estadísticas aparecen clasificados como "Top-Down" y por tanto como "tecno-economicistas", mientras aquellos discursos que enfatizan el comercio justo, los bienes públicos digitales, la inclusión y participación de comunidades fueron clasificados como "Bottom-Up" y en consecuencia como "socio-culturalistas".

Como consecuencia del análisis, no parece un argumento sólido que el "factor social" sea una especie de descubrimiento en alguna fase evolutiva del concepto "Smart City". Por el contrario, este estudio plantea que las diferentes narrativas se deben a interpretaciones desde encuadres 
determinados ideológicamente. Como se comenta en el apartado "Discusión", no parece tan casual que los planes de Estados neoliberales como Colombia y Chile promuevan políticas tecnocráticas ("tecno-economicistas"), que se advierten en el tratamiento del concepto "Smart City", mientras que organizaciones no gubernamentales 0 estudios académicos críticos promuevan narrativas más centradas en las personas ("socio-culturalistas").

Por lo tanto, habiendo obtenido estas dos narrativas predominantes, (denominadas tecno-economicista y socio-culturalista), en los siguientes sub-apartados se analizan los énfasis discursivos, lenguajes empleados, las ideas subyacentes, omisiones y categorías expresivas en el variado conjunto de textos que compone este trabajo.

\section{Acerca de la narrativa tec- no-economicista de Smart City}

En un artículo con el suspicaz título "Ciudades Inteligentes": ¿materialización de la sostenibilidad o estrategia económica del modelo neoliberal? las autoras Vásquez y Linares (2018) contraponen la idea de una materialización de la sustentabilidad respecto de una estrategia neoliberal sobre las "Smart City". Si bien, como veremos, no existe una dicotomía al respecto, puesto que la economía neoliberal sí tiene un discurso sobre el medioambiente (a veces llamado capitalismo verde), por ejemplo, a través de los derechos de emisión (ETS). Estos derechos se basan en una política de compensación de emisiones de C02, donde se intenta convertir el problema medioambiental en una externalidad que puede ser ingresada en la operación costo-beneficio. Así también, a través de la aplicación de nuevas tecnologías que reemplazan fuentes de energías contaminantes por energías renovables,
Ios problemas que la actividad productiva genera en el medio ambiente son atenuados mediante una renovada asociación con las nuevas tecnologías denominadas "Tics" ${ }^{3}$.

En esta versión, la inteligencia está asociada a la capacidad de gestión, la aptitud de acción de los individuos, al uso de tecnologías avanzadas, entre otras cualidades que hemos denominado como "tecno-economicistas". Reforzando esta idea "tecno-economicista" las propias autoras señalan que el propósito de las "Smart Cities" "se centra en lograr una gestión eficiente de los equipamientos urbanos que satisfaga las necesidades de los ciudadanos. Así, la innovación social acompañada de innovación tecnológica, se presentan como los principales motores de cambio" (Vásquez y Linares, 2018: 480). Así mismo, la Comisión Europea en su informe de 2012 establece que las ciudades y comunidades inteligentes son una asociación en las áreas de energía, transporte, información y comunicación, con el objetivo de catalizar el progreso de esas áreas (European Commission, 2012: 2).

Lo anterior revela la importancia central asignada a las tecnologías, las cuales tendrían el poder inherente de transformar la calidad de vida de las personas. Esta visión considera a los ciudadanos en cuanto consumidores (sistemas de personas), como los receptores de tecnologías que satisfarían sus necesidades, provistas por grandes empresas dedicadas a las "Tics" y la producción de energías renovables.

Esto último queda más claro cuando se revisa el documento Índice IESE "Cities in Motion" 2019, elaborado desde la perspectiva económica de la escuela de negocios de la Universidad de Navarra, donde se señala que las urbes "deben recurrir a la innovación con más frecuencia para mejorar la eficiencia y la sostenibilidad de sus servicios. Además, han de fomentar la comunicación y lograr que ciudadanos 
y empresas se impliquen en los proyectos" (Berrone y Ricart, 2019: 10). En esta plataforma se propone un marco conceptual donde se ha creado un índice que integra 9 dimensiones: capital humano, cohesión social, economía, gobernanza, medioambiente, movilidad y transporte, planificación urbana, proyección internacional y tecnología.

Sin pretender examinar exhaustivamente cada uno de los indicadores que componen estas 9 dimensiones, es importante señalar que para abordar el factor social se emplean términos como "capital humano y cohesión social", cuyas unidades de medida son: Proporción de la población con educación secundaria y superior; Número de escuelas de negocios; Movimiento internacional de estudiantes; Número de universidades de la ciudad que están en el top 50; Número de museos y galerías de arte por ciudad; Número de escuelas públicas o privadas por ciudad; Número de teatros por ciudad; Gasto en ocio y recreación per cápita; Gasto en ocio y recreación expresado en millones de dólares y Gasto en educación per cápita (Berrone y Ricart, 2019)., para medir el capital humano de una ciudad. Cabe destacar el carácter no sólo cuantitativo, sino el indicador utilizado: "museos" de una ciudad; escuelas de negocios o el número de universidades que están en el top 500. También es necesario notar que para el término "cohesión social" se utilizan indicadores tales como: Ratio de fallecimientos; Índice de criminalidad; Índice de sanidad; Tasa de desempleo; Índice de Gini; Precio de la propiedad; Ratio de mujeres trabajadoras en la administración pública; Índice de paz global; Índice de felicidad; Proporción de esclavitud; Terrorismo: Número de altercados vandálicos; Variable que mide si una ciudad brinda un entorno amigable para la mujer; Ratio de suicidios y Ratio de homicidios (Berrone y Ricart, 2019).
Lo anterior se repite con más o menos los mismos componentes en cualquier ejemplo de medición de "Ciudad Inteligente" que se busque bajo estos parámetros. Se encontrará la generalidad que se puede resumir en lo siguiente: "hace referencia a un uso extensivo y eficiente de las tecnologías disponibles -en particular las TIC- dirigidas a mejorar la calidad de vida de la población" (Alvarado, 2018: 2).

El reiterativo concepto de "calidad de vida" utilizado en esta versión de "Smart City" se transforma en una especie de significante articulador, alrededor del cual se tiende a concentrar la visión sobre el factor 0 componente social.

En general se advierte que el concepto de calidad de vida se utiliza en la esfera del individuo y la satisfacción de sus necesidades, como confort, seguridad y empleo, eximiendo cualquier indicador que asigne importancia a la pertenencia a grupos sociales extensivos 0 entidades de participación social.

Así también, se da por supuesto que el orden económico es el neoliberalismo en tanto ideología subyacente que se caracteriza por un rol subsidiario del Estado como actor económico, la privatización de toda la actividad económica, las reglas del mercado aplicadas a toda la esfera del comportamiento social, la libre circulación de mercancías y capitales, y el objetivo fundamental puesto esencialmente en el crecimiento económico, entre sus aspectos principales. Esto se advierte nítidamente en el Índice IESE "Cities in Motion" (Berrone y Ricart, 2020), donde en el componente economía algunos de sus indicadores enfatizan cuestiones como: Productividad laboral; Facilidad para comenzar un negocio; Número de empresas que cotizan en la bolsa; Proyección anual de crecimiento del "PIB", entre otros indicadores. En esta lógica no parece extraño que un indicador mida si la ciudad cuenta o no con servicio de "Uber" 
(norteamericana) y en otro indicador se mide si la ciudad cuenta o no con el servicio "Glovo" (española).

Por otra parte, resulta revelador que, en un componente importante como la Gobernanza, los indicadores midan las reservas en millones de dólares de una ciudad, el índice de percepción de la corrupción, o si el gobierno está o no utilizando tecnologías de la información. Si bien, por otra parte, un indicador mide el importante ranking de democracia, lo hace, no obstante, a través de la información proporcionada por "The Economist", un prestigioso semanario representante del liberalismo económico extremo, que evidencia una posición enunciativa.

Los gobiernos de Chile y Colombia han implementado políticas de gobierno digital que incluyen "Smart City". En el caso chileno, en este estudio se han revisado varios documentos relacionados, entre ellos el Diagnóstico, levantamiento de brechas e identificación de oportunidades del Gobierno de Chile y la Fundación País Digital y la Fundación Fraunhofer dirigida principalmente al estudio de industrias inteligentes, donde se señala que:

La transición de Chile hacia una industria inteligente requiere observar como otros países líderes abordaron este objetivo. Las principales buenas prácticas observadas incluyen (a) definir visiones e identificar oportunidades; (b) priorizar recursos; (c) fijar el nivel de competitividad; (d) avanzar en la gestión de cambio del Estado y las empresas; (e) fomentar las I+D ${ }^{4}$; (f) impulsar cultura de colaboración público-privada; $(\mathrm{g})$ crear políticas de fomento; (h) operativizar de lo estratégico a lo táctico (CORF0, 2015).

Quedan serias dudas si la mayoría de estas prácticas no son prácticas ya habituales de las empresas y el calificativo de empresas inteligentes es casi decorativo. Resulta notoria la ausencia de discusión relativa a políticas laborales y las relaciones con las asociaciones de trabajadores, para el cual, el documento se refiere más adelante como "capital humano especializado". Las escasas veces que se cita el término "trabajador" se refiere a su reemplazo por tecnologías robóticas, su escasa especialización 0 problemas vinculados a seguridad laboral. En el capítulo dedicado a las Smart Cities se las define de la siguiente manera:

Las Smart Cities son ciudades que, por medio de las aplicaciones de la tecnología en sus diferentes ámbitos, se transforman en localidades más eficientes en el uso de los recursos disponibles. Ejemplos de los beneficios son el ahorro y la mejor utilización de la energía, las mejoras de los servicios entregados a la población y la promoción de un desarrollo sustentable con el medio ambiente. (CORF0, 2015: 49)

En el cuadro denominado "Anatomía de las Smart Cities" en la línea de ejemplos de participantes, sólo aparecen logotipos de grandes empresas como "Movistar, Entel, Claro, Esval, Esbio, Aguas Andinas, Chilectra, Lan" y empresas forestales, entre otras. En la línea dedicada a la Unidad Coordinadora en sus distintas escalas sólo aparecen las Juntas de Vecinos entre varias reparticiones estatales. Para confirmar la importancia de las "Tics" en el modelo chileno, se destaca el documento "Digital Government in Chile: Strengthening the Institutional and Governance Framework", que promueve la relación entre las tecnologías digitales y la gobernanza institucional, proporcionando información que contribuye al debate sobre la gobernanza de las tecnologías digitales en el sector público y sobre el diseño de entornos institucionales que generen impacto (OECD, 2016).

En el caso del gobierno digital de Colombia se examinaron varios antecedentes, entre ellos, el documento Propuesta de un Modelo 
de Medición de Madurez de Ciudades y territorios inteligentes para Colombia, en el apartado de abril de 2020 señala que: El análisis de diferentes modelos de "ciudades inteligentes" evidenció que, si bien existen diferentes definiciones de "ciudad inteligente", en su gran mayoría convergen hacia un concepto más amplio y ambicioso que el simple fortalecimiento de una dimensión digital de ciudad, y proponen un alcance mayor con relación a conceptos tales como la sostenibilidad, la eficiencia, la participación ciudadana, la innovación, la gobernanza y la inclusión social. Todo ello aplicado a los diferentes ámbitos de la cotidianidad de cualquier ciudad como: "la eficiencia energética, la ordenación urbanística, la movilidad, el medio ambiente, la seguridad ciudadana, la economía circular, el turismo sostenible, la generación de valor de ciudad, entre otros". (MINTIC, 2020ª 6).

En otro documento del gobierno colombiano, documento de recomendaciones para el desarrollo de ciudades y territorios inteligentes (MINTIC, 2020b) los autores se preguntan ¿Qué es un modelo de madurez?, y se responde con la siguiente definición:

Un modelo de madurez es una herramienta que permite identificar Ios niveles a través de los cuales una persona, organización o ciudad avanza hacia la realización de un objetivo final, permitiendo medir ese proceso continuamente y estableciendo desde el inicio una hoja de ruta para llegar al estado 0 etapa deseada. (MINTIC, 2020c: 40)

Para los autores del documento colombiano, "resulta ser una herramienta muy útil en materia de ciudades y territorios inteligentes [...], dado que estas iniciativas se planifican a mediano y largo plazo, se requiere de la generación de indicadores específicos que permitan orientarlas de forma acertada" (MINTIC, 2020c: 40).
Entre las características del Modelo de

Madurez se establecen algunas premisas como "Primero el ciudadano", el cual, tendría acceso a servicios públicos confiables y de calidad, así como la satisfacción de sus necesidades de forma inteligente e integral. También se indica elementos como la inclusión y transparencia en la comunicación con los ciudadanos; y otras premisas de orden técnico como la generación de valor en la adquisición de infraestructura adecuada y la eficiencia en el uso de los recursos. Si bien existen variables sociales en este modelo, se trata en lo sustantivo de encuestas realizadas a la población, donde la ciudadanía es entendida como destinatario de servicios estatales, como beneficiaria de asistencias sociales (de salud, por ejemplo), así como receptor de formas de comunicación más inclusivas.

Todas estas premisas se enmarcan en los límites de lo que se entiende como "calidad de vida", en el mismo documento se define como "los aspectos que faciliten y favorezcan la interacción e inclusión segura entre las personas y los entornos económicos, sociales, de salud y bienestar, entre otros, para satisfacer de manera adecuada y satisfactoria las necesidades de las personas" (MINTIC, 2020c: 42). Esto se realiza a través de una forma de relación política con los ciudadanos denominada "Gobernanza", que "comprende la política pública, los procesos y los mecanismos que permitan la interacción y la participación segura entre gobernantes y gobernados de tal forma que favorezcan la toma de decisiones y una mayor eficiencia, transparencia y colaboración" (MINTIC, 2020c: 41). En las conclusiones del documento se señala que "El modelo aplicable a "ciudades inteligentes" no debe corresponder a una ciudad híper-tecnológica. Añadir tecnología a una ciudad no la hace inteligente" (MINTIC, 2020c: 53), no obstante, resulta contradictorio, pues los factores prácticamente son todos tecnológicos ya 
que cuando se caracteriza a una ciudad inteligente se sostiene que:

Una ciudad inteligente se caracteriza por ser una ciudad con una visión propia de sí misma para dar solución a sus problemas (el desarrollo sostenible, la infraestructura, el medio ambiente, la economía digital, la transformación digital, la tele salud, la telemedicina, la tele educación, la movilidad inteligente, la seguridad ciudadana, la economía circular, las energías renovables, el turismo sostenible, entre otros), centrándose en generar valor y mejora en la calidad de vida de la ciudadanía. (MINTIC, 2020c: 53)

\section{Acerca de la narrativa socio-culturalista de Smart City}

La acepción economicista y tecnocrática del concepto "Smart City" tiene detractores y no faltan quienes consideran que se trata de una visión o etapa superada en el desarrollo del concepto, esto resulta difícil de sostener pues nada indica que las tendencias que conciben las "Smart Cities" desde un modelo de economías basadas en el predominio pleno del libre mercado se encuentre en retirada. Incluso se sostiene que existirían tres fases en el desarrollo de la "Smart City", como señala Cohen (2015), estas fases comenzarían por una liderada por las empresas, pasando por los gobiernos, hasta una última fase impulsada por los ciudadanos. Tal como se ha indicado precedentemente, el factor social o ciudadano no se encontraba ausente, sino más bien invisibilizado.

En todo caso en forma creciente se exponen planteamientos que disputan los contenidos 0 el orden de prioridades de preocupaciones de una "Smart City". En un breve artículo Ray Lara llama la atención sobre el caso de Tequila (México) como destino turístico inteligente, para lo cual enfatiza la idea de que son territorios con imagen e identidad propia (Lara, 2017: 2), es así como en el año 2013 se crea el Consejo para el Desarrollo Integral de Tequila (CODIT) que funciona como Organismo Gestor del Destino, y según Lara (2017) se basa en el modelo de cuádruple hélice, así en el año 2014 junto con el Ayuntamiento de Tequila se presenta ante el Programa de Pueblos Mágicos el proyecto Tequila Pueblo Mágico Inteligente (:11). El modelo de cuádruple hélice que se cita, es la integración de la sociedad a un modelo que anteriormente se conocía como triple hélice (Administración, Investigación, Empresa).

Este nuevo modelo, según se indica, integra en forma más decidida a las personas y es una tendencia más transversal de colaboración y participación ciudadana, en la cual, los expertos aportan su conocimiento y los ciudadanos su conocimiento local y experiencial, dado que se supone conocen mejor el estado de un territorio. Sin embargo, no parece convincente que modelos urbanos no consideren el factor social, sino que más bien su omisión (triple hélice) indica una forma excluyente de tratar con lo social.

Un ejemplo de este paradigma social es el programa de desarrollo para la ciudad de Tequila en Jalisco, México, donde hemos revisado los documentos de la Fundación Beckmann, que en su memoria anual 2020 indica:

...Todo este esfuerzo es para asegurar que perduren los recursos a través del tiempo y las familias puedan salir adelante ante cualquier circunstancia [...] ¿Y al final, qué se desea?: el bienestar de las personas. Nuestra misión es contribuir al desarrollo y a la mejora de la comunidad de Tequila, apoyando a través de la educación, capacitación y cultura a elevar su calidad de vida, rescatando su patrimonio tangible e intangible, de tal forma que 
destaque el valor del destino turístico.

(Fundación Beckmann, 2020: 24)

Mediante un modelo de innovación social y economía solidaria con ecosistemas de apoyo se ha podido transformar esta pequeña localidad de poco más de 40.000 habitantes en un ejemplo para otras ciudades de México y latinoamérica. A través de iniciativas como el primer Centro de Innovación Social en Tequila inaugurado en septiembre de 2019 que permitió ofrecer una alternativa de formación de manera remota a muchos jóvenes que por la crisis sanitaria no pudieron seguir con sus estudios. Este modelo se presentó en el seminario "Valdivia Urban Lab" (Chile) que comentaremos a continuación.

El reciente evento denominado "Valdivia Urban Lab", 2021 desarrollado entre los días 27 y 8 de mayo en formato virtual, nos permite presentar varios planteamientos de esta narrativa socio-culturalista. A través, de varias ponencias que comentaremos, el evento ha expuesto casos y argumentos donde la diferencia social es abordada mediante conceptos como desigualdad social, actores sociales 0 experiencias comunitarias y las tecnologías son consideradas como factores colaborativos.

En este mismo evento, las expositoras Sonia Espínola y Margarita Arana presentaron la ponencia Construcción de redes comunitarias en "ciudades inteligentes", donde según señalaron se busca un modelo de crecimiento integral y sostenible para el pueblo de Tequila en Jalisco, denominado "Tequila Inteligente", a través de la colaboración entre actores sociales, económicos y culturales (Espínola y Arana, 2021). Esto, según lo expresado, ha posicionando a la ciudad como una ciudad patrimonial a través de la difusión de las riquezas culturales y naturales de la región, así como la promoción de la economía local y el fortalecimiento de la comunidad a partir de experiencias culturales comunitarias
(Espínola y Arana, 2021).

Efectivamente la iniciativa tiene como centro el desarrollo humano de comunidades vulnerables y pequeños artesanos mediante lo que llaman "ecosistemas de apoyo", inscrito en un modelo integral social. De acuerdo a sus informes la pobreza decreció un $61 \%$.

La ponencia de Paula Roldán con el tema ¿Qué implica ser una "Smart City" en América Latina?, la expositora de "Ruta N Medellín", Colombia, afirma que una ciudad inteligente es aquella que maximiza la inclusión y la igualdad social al tiempo que mejora la prestación de servicios (Roldán, 2021), también señaló que una estrategia de "ciudades inteligentes" no es necesariamente una estrategia de desarrollo económico, es una estrategia de mejoramiento de la calidad de vida (Roldán, 2021) , lo cual implica una definición distinta de calidad de vida a la revisada precedentemente, para esta expositora la innovación es un proceso colaborativo con el ciudadano al centro del proyecto.

En este mismo evento, Pontus Westerberg representante de "UN-Hábitat for a better urban future" (programa de las Naciones Unidas para los asentamientos humanos) en su exposición denominada "Ciudades Inteligentes" para la ciudadanía, señaló que había 4 defectos de la visión de la ciudad inteligente:

1. Comenzar con tecnología en lugar de desafíos urbanos.

2. Uso o generación de evidencia suficiente.

3. Falta de conciencia de cómo otros están trabajando para mejorar las ciudades.

4. Insuficiente participación ciudadana y de las partes interesadas.

Para superar este diagnóstico Westerberg propone, entre otros principios: Centrar las actividades de las "ciudades inteligentes" 
en las necesidades de las personas; Adoptar un enfoque colaborativo; Construir una base de acceso universal a internet; Desarrollar la capacidad digital interna de las personas de una ciudad; Maximizar la participación, representación y control de la comunidad (Westerberg, 2021). Resulta muy llamativo e interesante los principios que propone, Derechos digitales a través de la participación por diseño o nociones como "la gobernanza de datos" que incluye la rendición de cuentas y la inclusión social, asi como los bienes públicos digitales, para que sean abiertos, transparentes, accesibles e interoperables. Es decir, un acceso universal e igualitario a internet, así como la completa alfabetización digital.

La ponencia con la cual concluyó el evento fue la exposición de Alfonso Jiménez Lara, de la megaempresa de telecomunicaciones china "Huawei", con la ponencia "Desarrolla territorios inteligentes con Huawei". El expositor, entre otros temas, exhibió un video denominado "El guardián en el cielo", el cual muestra la vida de campesinos chinos en su actividad rural dedicada a la agricultura muy tradicional del cultivo arrozales, (Luxi County, Yunnan, China). Todo el fatigoso trabajo depende de condiciones climáticas impredecibles para ellos, "está fuera de nuestras manos, es el destino", según dicen. La naturaleza tiene, para estos campesinos, una mente propia que no pueden conocer con anticipación y a veces los cultivos no crecen debido a los fuertes vientos 0 ataques de insectos. Pero con los satélites "Gaofen" puestos en órbita por China, especialmente "Gaofen-6" dedicado a registrar el contenido de cultivos de la tierra, que pueden observar la geología y el suelo del área, permite entregar consejos concretos a los agricultores para que puedan tomar decisiones acertadas en el manejo de sus campos. Hoy estos campesinos cuentan con "lap-top", que en tiempo real reciben valiosa información a través de la plataforma abierta de intercambio de datos CNSA GEO (con la ayuda de "Huawei Cloud, Geovis, Piesat y Cresda") en interfaces que pueden ser leídas fácilmente por los campesinos. La información satelital, según se indica en el video, está siendo enviada en forma gratuita también a agricultores pobres de otros puntos del planeta, incluida Latinoamérica.

En todos los ejemplos de esta sección se advierte un lenguaje inclusivo como: desarrollo integral; innovación social; experiencias comunitarias; igualdad social; control de la comunidad; derechos digitales; bienes públicos digitales, etc. así como experiencias concretas que ejemplifican al menos sensibilidades alternativas. Si bien se trata de pequeñas localidades 0 actividades focalizadas, constituyen sin duda aportes interesantes al debate sobre las "Smart Cities".

\section{Acerca de narrativas emergentes eco-utópicas.}

Es interesante mencionar en este estudio propuestas recientes (al menos en Latinoamérica) respecto de las posibilidades de abordar una "Smart City". Se trata de iniciativas que no se aplican a ciudades o localidades existentes, sino en emplazamientos vírgenes que inauguran una realidad nueva. Su particular carácter, del cual no se tienen mayores antecedentes que aquellos propios de proyectos urbanísticos y promesas ideales, ha sido el motivo por el cual no se ha incluido en este estudio en forma analítica, no obstante, resulta interesante para futuros análisis del fenómeno, que por ahora se ha preferido caracterizar como eco-utópico. Un ejemplo es la "Ciudad-bosque" del arquitecto Stefano Boeri (Boeri Architetti Studio) para Cancún, México, proyecto para 130.000 habitantes en 557 hectáreas y según el arquitecto forma parte de un nuevo paradigma en la forma de pensar las ciudades del futuro (Martin, 2019). Boeri también tiene proyectos similares en otras partes del mundo. 


\section{Discusión}

El análisis anterior permite formular las siguientes consideraciones a modo de discusión:

\section{Polisemia del concepto Smart City}

Como se ha argumentado el concepto "Smart City" no es un concepto cerrado, sino que es utilizado bajo diferentes modalidades enunciativas y para propósitos diversos.

La noción de inteligencia es asumida desde diferentes ángulos, desde la idea de realidad como operatividad de datos cuantificables, la inteligencia como gestión eficiente con criterios de sustentabilidad o la inteligencia como colaboración entre procesos maquínicos y actividades humanas cooperativas, según se trate del lugar de enunciación.

Si bien existe una constante enmarcada en los componentes tecnológicos y eco-sustentables, el concepto "Smart City" fluctúa entre el énfasis dado a los componentes de gestión de empresas y la gobernabilidad centralizada, por una parte, hasta la participación ciudadana y los bienes públicos digitales por otra. Esto es lo que ha proporcionado la argumentación para proponer al menos dos narrativas predominantes del concepto "Smart City".

\section{El factor social}

En la narrativa "tecno-economicista" el sujeto es concebido en términos abstractos como "capital humano" y receptor 0 consumidor de tecnologías, pero exento de una dimensión social autónoma 0 emancipada, ya que siempre depende de la asistencia o control gubernamental. Otro aspecto interesante es el énfasis en la seguridad pública como un problema agudo y un elemento connatural en la convivencia social en la realidad de estos países, como algo que se integra en la ecuación, pero que no tiene solución. Se puede afirmar que el término "inteligente" en esta lógica, se concibe más como las posibilidades entregadas por las tecnologías de la información en la gestión, dirección y administración de la ciudad, que en términos de mayor igualdad social o económica.

En esta versión se advierte una tendencia al uso de protocolos cuantitativos y competitivos. Si bien el uso extendido de indicadores cuantitativos puede sostenerse en la premisa de que las mediciones y datos serían la mejor forma de abordar seriamente estudios de población, se puede rebatir, por ejemplo, que el índice "per cápita” no entrega información importante para consideraciones sobre distribución del ingreso porque distorsiona groseramente la realidad en términos matemáticos.

Cabe señalar que en ciertas formas de medir la realidad (caso de IESE "Cities in Motion" 2019, de Berrone y Ricart), como el grado de consenso de los miembros de un grupo social y el nivel de convivencia entre los conjuntos de personas diferente, no contenga indicadores que midan 0 permitan analizar la participación ciudadana, la afiliación a sindicatos, la existencia de organizaciones sociales o territoriales, etc. y en cambio 5 de 16 indicadores enfaticen aquellos elementos que están relacionados con problemas de seguridad pública como el índice de criminalidad, el índice de paz global (que mide el nivel de paz y la ausencia de violencia en un país o región), el ratio de homicidios, el número de altercados vandálicos y el ratio de homicidios.

En la narrativa denominada "socioculturalista" el sujeto ya no es concebido como un individuo aislado y consumista, sino como comunidades territoriales que presentan trastornos sociales, pero también oportunidades. Así y todo, no hemos encontrado en la revisión realizada en la versión "socio-culturalista" debates 
y propuestas en torno a cuestiones como el derecho a la ciudad, la noción de bienes comunes 0 el régimen de propiedad, por ejemplo. No se advierten postulados alternativos a las formas de producción y reproducción del capital bajo el régimen de producción capitalista en la realidad latinoamericana, ya que si bien proyectos locales parecen otorgar efectivamente mejores y más justas condiciones de vida, el que puedan funcionar en pequeñas escalas no nos dice nada acerca de que puedan replicarse en escalas extensas de ciudades metropolitanas con millones de habitantes 0 en escalas de ciudades intermedias.

Cabe entonces preguntarse si esta narrativa se corresponde con un modelo de tipo asistencialista o paternalista respecto de las comunidades que aborda.

\section{Carácter ideológico de las narrativas}

Tal como se ha argumentado precedentemente, la polisemia de significados del concepto "Smart City" se tiende a explicar generalmente por una especie de evolución natural del concepto en el tiempo, mientras este estudio sostiene la idea que se debe considerar más bien como énfasis enunciativos de carácter ideológico.

Este carácter ideológico que se propone aquí se configura en los contornos geopolíticos de la región, dentro de parámetros y lineamientos que forman parte del sistema capitalista global.

Por ejemplo, resulta sorprendente que en IESE "Cities in Motion" 2019, (Berrone y Ricart) la dimensión Proyección Internacional, se mida según el número de establecimientos de la cadena "McDonalds" por ciudad o el ranking de ciudades según el número de fotos tomadas en ellas y subidas a "Panoramio" o el índice de los precios de comidas y bebidas en restaurantes y bares en comparación con la ciudad de
Nueva York mientras que en la dimensión 9 sobre Indicadores de tecnología se mide la cantidad de miembros de "Linkedin". Esto presenta un sesgo que podríamos denominar como neocolonial, donde los parámetros para medir ciudades del mundo sub-desarrollado en Latinoamérica corresponden a multinacionales, transnacionales del mundo avanzado 0 ciudades norteamericanas $\mathrm{y} / 0$ europeas.

Políticas de Estado, como las de Chile y Colombia son concordantes con los modelos económicos neoliberales sostenidos por estos países en las últimas décadas y su relación con los discursos emanados en sociedades desarrolladas, como son EEUU y Europa. Dicha cuestión se advierte, como se ha dicho, en las persistentes referencias a normas 0 estándares utilizados en estos países y a la reiterada cita a la ciudad de Nueva York como parámetro de calidad de vida. El establecimiento de universidades europeas como generadoras de discurso y la implementación de tecnologías producidas por países avanzados, son todas cuestiones que van incrementando una visión que evita abordar temáticas tales como la desigualdad social o el conflicto social y son reemplazadas por nociones vagas de calidad de vida o cohesión social. Se puede apreciar, que los indicadores utilizados son en su mayoría de orden cuantitativo y el fomento a la innovación se erige por medio de rankings de competitividad. La percepción de felicidad ciudadana, es medida a través de encuestas y la educación digital es más la construcción de un discurso motivador, que una transformación real para impactar los territorios.

Una cuestión importante que se deriva del análisis de esta versión, es que el énfasis otorgado a los recursos cuantitativos, las técnicas de encuestas, la importancia dada a los rankings, el fomento a la competitividad y la eficiencia, así como las referencias de las potencias económicas híper-desarrolladas, 
utilizadas como criterios de evaluación, en ausencia de otros factores y herramientas de análisis, implican ya una tecnificación de la realidad y suponen una especie de idealización donde el conflicto social tiende a estar completamente ausente.

Por otra parte, la narrativa "socioculturalista" se ve confirmada en el ámbito de discursos focalizados en organizaciones no gubernamentales u organizaciones aliadas con la empresa privada, como es el caso mexicano de la Fundación Beckmann, la empresa productora de "Tequila Cuervo" y en proyectos de impacto local como la ciudad de Tequila en Jalisco. En esta versión existe un cambio en el lenguaje y el lugar que ocupa en la cadena significante del discurso el medio tecnológico, desplazado por orientaciones de tipo social. Sin embargo, es posible que estas iniciativas podrían incluirse en lo que se denomina como responsabilidad social empresarial y apoyo a proyectos de pymes o microemprendedores locales.

En tal caso cabe preguntarse si tales iniciativas corresponden a versiones alternativas de un capitalismo con rostro humano, o son simplemente estrategias al interior del propio modelo de capitalismo neoliberal global, que desplaza una parte de la fuerza de trabajo hacia iniciativas de trabajo autónomo.

\section{¿Otras posibilidades?}

De lo anterior se deriva la pregunta por posibilidad de evolución del concepto y su utilidad en prácticas sociales más allá de los contornos estado-céntricos o corporativos. Consideramos que las narrativas "tecnoeconomicistas" y "socio-culturalistas" se inscriben en los contornos económicos de la forma de producción capitalista, desde la versión neoliberal más acentuada hasta posiblemente una versión reformista de un capitalismo "sin fricciones" ${ }^{5}$. Sin embargo,
City" no pueda ser replanteado en el contexto de otras formas de concebir las relaciones sociales en la ciudad latinoamericana, donde la inteligencia puede llegar a entenderse como "inteligencia social" o más aún como señala Cooper (Cooper, 2017) la necesidad de politizar el concepto, convencer a políticos y líderes, generar metodologías participativas y lineamientos políticos transversales.

Esto, sin embargo, exigiría la formulación de nuevo tipo de indicadores más apropiados a la realidad de los países en desarrollo, que no dependan de los estándares y realidades de los países desarrollados. Por esto, nos parece que el término "Smart City" no está agotado ni circunscrito al marco socioeconómico analizado, sino todavía permanece abierto a nuevos contenidos desde ámbitos críticos.

La tecnología, puede efectivamente (y de hecho lo hace) ayudar a mejorar las condiciones de vida de las personas, pero no opera en condiciones abstractas, sino en condiciones reales y así como la educación, la salud o la previsión constituyen espacios de desigualdad social en la realidad latinoamericana, la tecnología también podría ser (y de hecho lo es) un espacio de desigualdad, de aumento de la brecha social y también un dispositivo de dominación y control. La tecnología como el diseño, no tienen la capacidad de resolver el conflicto político y la colisión social que constituye una sociedad con extremas diferencias de clases sociales originadas en las brechas de desigualdad social, cuestión que es inherente a la relación entre los seres humanos y no a la relación con las cosas. Por ejemplo, en el modelo "Smart City" tal como está planteado existe una ausencia respecto de problemas cruciales de la producción del espacio urbano, por ejemplo, procesos anómalos como la segregación urbana o la marginalidad social de los enormes guetos 0 "slums" de las ciudades latinoamericanas. 
En cuanto a la dimensión medioambiental es vital para la sustentabilidad de la vida urbana, pero sólo tiene alguna factibilidad sobre la base de considerar el ecosistema entre los bienes comunes colectivos (0strom, 1990), es decir como un factor que debe estar al centro de la discusión. En función de lo anterior, es posible sostener que una ciudad inteligente en un territorio inteligente debería supeditar el interés individual a los intereses colectivos y los equilibrios eco-sistémicos. Una ciudad que se mide no a sí misma, sino en la relación activa de las comunidades y el medio ambiente.

Pero esto devuelve la discusión al ámbito de la política y el concepto de democracia, a las que están supeditadas las tecnologías y sus usos. Esto exige un modelo de democracia menos representativa y más activa, transparente y colaborativa donde son las propias comunidades implicadas quienes mejor pueden gestionar su entorno, como lo aborda la idea de bienes comunes de Ostrom.

Sin embargo, lejos de impugnar el concepto, este estudio está a favor de reconvertir su contenido en función de una visión que la sitúe en el contexto y realidad latinoamericana.

Una política en Latinoamérica centrada en la "diferencia social", es decir, en la enorme y compleja multiplicidad de "eco-sociosistemas" en Latinoamérica, desde las comunidades mayas de Centroamérica, los pueblos indígenas de Ecuador y Brasil, los campesinos de la coca en Bolivia, las extensas comunidades mapuche en Chile y Argentina, como también las megalópolis de Ciudad de México, Sao Paulo, el gran Buenos Aires o Santiago de Chile, que forman una misma región con los sistemas ecológicos de la selva maya, la selva amazónica, los bosques tropicales o el bosque profundo del sur de Chile. Este cosmos multifacético que está atravesado por extremas diferencias de clases sociales, enclaves de narcoterrorismo comunidades indígenas desmembradas y perseguidas, violencia urbana sostenida, pobreza endémica y zonas de sacrificio, entre otros males, no puede considerarse inmutable, ni mucho menos inteligente.

Como se advierte, se necesitan otras conceptualizaciones que permitan enriquecer el debate en torno a la idea de inteligencia de nuestras ciudades, conceptos como el derecho a la ciudad (Lefebvre, 2017), el de bienes comunes, elaborado por Elinor Ostrom (1990) o el de la propiedad del suelo como capital ficticio debatido convincentemente por David Harvey (2014), puede contribuir a una mejor reflexión sobre el futuro de las ciudades latinoamericanas. Sin embargo, nada de esto es puesto en debate en ninguna de las dos versiones revisadas en nuestro análisis.

El debate en torno a la inteligencia de nuestros territorios no puede zanjarse aquí. Estos análisis son sólo un aporte para abrir la discusión a los estudios urbanos en general y a las disciplinas afines, que tratan el espacio y sus dinámicas. La utilización de documentos gubernamentales y la discusión en torno al término, no son la única variable que permita identificar discursos y lógicas totalizantes en torno al término "Smart City" en la producción científica. Sin embargo, constituyen una forma de entrada que puede contribuir al debate sobre el uso de la noción de inteligencia de nuestras ciudades y posibilitar explorar otras miradas en torno a la capacidad de aprender de nuestros territorios. La "Smart City" surge, así como una oportunidad para refrescar las metodologías de la producción espacial latinoamericana y posibilita desafíos nuevos para los futuros proyectos urbanos de nuestra región. Es temprano aún para decidir si proyectos como los de Stefano Boeri (denominado como "eco-utópico") emerjan, 0 deban considerarse, como alternativas liberadoras 0 emancipadoras. 
Otra cuestión que queda en evidencia en base a los resultados obtenidos, es que lejos de alejarnos de un momento tecnocrático en el uso del término y avanzar hacia uno socio cultural, las narrativas "tecno-economicista" y "socio-culturalista" compiten e incrementan su utilización desde un mismo tablero de juego. Es decir, los modos de apropiación del término no va acompañado de reflexiones epistemológicas sobre dicho uso, por lo que aparece un momento de oportunidad a la investigación en pos de una construcción conceptual espacial en nuestra realidad latinoamericana. 


\section{Referencias:}

Alvarado, R. A. (2018). Ciudad inteligente y sostenible: hacia un modelo de innovación inclusiva TT - Smart and Sustainable City: Towards an inclusive innovation model. PAAKAT: revista de tecnología y sociedad, 7(13). https://doi.org/10.32870/ pk.a7n13.299

Bauer, M., Helbig, D., Mokhov, V., Eltsova, M. (2019) J. Smart Region concept as a solution for sustainable development for region with a rural and urban character Journal of Physics: Conference Series, Volume 1415, International Conference on Innovation Energy 2-3 0ctober 2019, Perm, Russian Federation. Recuperado en: https://iopscience.iop.org/ article/10.1088/1742-6596/1415/1/012018.

Berrone, P. y Ricart, J. (2019) "Índice lese: Cities In Motion". Universidad De Navarra, España.

Cohen, B. (2015). The 3 Generations of Smart Cities. Recuperado en: https:// www.fastcompany.com/3047795/the-3generations-of-smart-cities

Cooper, D. (2017). Smart Cities en Chile: Entre la factibilidad tecnológica y las barreras políticas . Debates Latinoamericanos, 31, 10-19.

CORF0 (2015). Diagnóstico, levantamiento de brechas e identificación de oportunidades. Programa Estratégico Industrias Inteligentes. Chile. Recuperado en: http://seguimiento. agendadigital.gob.cl.

Espínola, S y Arana, M. (2021). Construcción de redes comunitarias en ciudades inteligentes. Ponencia presentada en Valdivia Urban Lab 2021. Valdivia, Chile. Recuperado en https://www.valdiviaurbanlab.cl.
European Commission. (2012). Smart Cities and Communities. Recuperado en de The European Innovation Partnership on Smart Cities and Communities: http://ec.europa.eu/ eip/smartcities

Fundación Beckmann (2020). Informe anual, 2020. México.

Harvey, D. (2014) Ciudades rebeldes. Del derecho de la ciudad a la revolución urbana. Madariaga, Juanmari. Madrid: Akal.238.

Jiménez, A. (2021) Desarrolla territorios inteligentes con Huawei. Ponencia presentada en Valdivia Urban Lab 2021. Valdivia, Chile. Recuperado en https://www. valdiviaurbanlab.cl.

Lara, R. (2017). Lo smart como dispositivo de atractividad territorial. Revisión del caso de Tequila como destino turístico inteligente. Debates Latinoamericanos, 31, 26-40.

Lefebvre, H. (2017). El derecho a la ciudad. Capitan Swing Libros, S. L.

Linares, J. y Vásquez, K. (2018). Ciudades inteligentes: ¿materialización de la sostenibilidad o estrategia económica del modelo neoliberal?. El Ágora USB, 18(2), 479-495. Recuperado en: https://doi. org/10.21500/16578031.3134

Martin, C. (2019) ¿Cómo serán las ciudades del futuro? Stefano Boeri prepara las primeras en Holanda y Cancún. Revista AD. Recuperado en https://www.revistaad.es/ arquitectura/articulos/como-seran-ciudadesfuturo-stefano-boeri-prepara-primerasholanda-y-cancun/24228

MINTIC (abril 2020) Propuesta de un Modelo de Medición de Madurez de Ciudades y Territorios Inteligentes para Colombia (MMMCTIC). Colombia. Recuperado en: https://estrategia.gobiernoenlinea.gov.co. 
MINTIC (octubre 2020) Documento de recomendaciones para el desarrollo de ciudades y territorios inteligentes (DRDCTI). Colombia. Recuperado en: https://estrategia. gobiernoenlinea.gov.co.

OECD (2016), Digital Government in Chile: Strengthening the Institutional and Governance Framework, OECD Digital Government Studies, OECD Publishing, Paris. Recuperado en: http://dx.doi. org/10.1787/9789264258013.

Orgaz, C. (2021) Cómo es Songdo, la ciudad inteligente creada desde cero en Corea del Sur. BBC News. https://www.bbc.com/ mundo/noticias-57030345

Ostrom, E. (1990) Governing the Commons: The Evolution of Institutions for Collective Action. Cambridge University Press.

Roldán, P. (2021). ¿Que implica ser una Smart City en América Latina? Ponencia presentada en Valdivia Urban Lab 2021. Valdivia, Chile. Recuperado en https://www. valdiviaurbanlab.cl/

Saunders, T. y Baeck, P. (2015). Rethinking smart cities from the ground up. NESTA. Recuperado https://media.nesta.org.uk/ documents/rethinking_smart_cities_from_ the_ground_up_2015.pdf

UN- HABITAT. (2020) World Cities Report 2020. The Value of Sustainable Urbanization Recuperado en: https://unhabitat.org/ World\%20Cities\%20Report\%202020.

Westerberg, P. (2021). Ciudades inteligentes para la ciudadanía. Ponencia presentada en Valdivia Urban Lab 2021. Valdivia, Chile. Recuperado en https://www. valdiviaurbanlab.cl/ 